Original paper

\title{
A systematic review and meta-analysis of evaluation of serum interleukin 8 levels in hepatocellular carcinoma
}

\author{
Ebrahim Shakiba', Masoud Sadeghi ${ }^{2,3}$, Mohammad Shakiba ${ }^{3}$ \\ 'Department of Clinical Biochemistry, Kermanshah University of Medical Sciences, Kermanshah, Iran \\ ${ }^{2}$ Medical Biology Research Center, Kermanshah University of Medical Sciences, Kermanshah, Iran \\ ${ }^{3}$ Students Research Committee, Kermanshah University of Medical Sciences, Kermanshah, Iran
}

\begin{abstract}
Aim of the study: To estimate serum interleukin 8 (IL-8) level in patients with hepatocellular carcinoma (HCC) compared to controls and patients with chronic hepatitis (CH) and liver cirrhosis (LC).

Material and methods: Three databases, i.e. PubMed, Web of Science, and Scopus, were searched up to November 2017 without language restriction. The mean difference (MD) and 95\% confidence interval (CI) were used by a random-effects analysis in RevMan version 5.3 , and sensitivity analysis was performed as the secondary analysis.

Results: Out of 239 studies found, 10 studies recruiting $659 \mathrm{HCC}$ patients, 237 controls, 357 patients with LC, and 48 patients with $\mathrm{CH}$ were included and analyzed in the meta-analysis. The pooled MDs were $39.48(95 \% \mathrm{Cl}$ : 152.31, 406.47, $p<0.00001), 21.32$ (95\% Cl: $-6.04,48.68, p=0.13$ ), and 36.46 (95\% Cl: 21.77, 51.15, $p<0.00001)$ in the patients with $\mathrm{HCC}$ compared to the controls, the patients with $\mathrm{LC}$ and those with $\mathrm{CH}$, respectively.

Conclusions: An elevated serum IL-8 level in the HCC patients compared to the three other groups showed an increased risk for this cytokine in $\mathrm{HCC}$ patients. Therefore, this interleukin can be used as a new biomarker replacing alpha-fetoprotein (AFP) or as a clinical assay for evaluation of the pathogenesis and probably the progression or development of HCC.
\end{abstract}

Key words: hepatocellular carcinoma, chronic hepatitis, liver cirrhosis, cytokine.

\section{Address for correspondence}

Masoud Sadeghi, MSc, Medical Biology Research Center, Kermanshah University of Medical Sciences, Kermanshah; Students Research Committee, Kermanshah University of Medical Sciences, Kermanshah, Iran, e-mail: sadeghi_mbrc@yahoo.com

\section{Introduction}

Hepatocellular carcinoma is the sixth most common malignancy in the world and the third cancer in terms of mortality, whose treatment, diagnosis, and control are still a major concern [1]. This cancer is sometimes accompanied by chronic liver diseases such as hepatitis (hepatitis B virus [HBV] or hepatitis $\mathrm{C}$ virus [HCV] infection) [2]. The presence of the interleukin 8 (IL-8) gene in a laboratory environment suggests that ILs can contribute to the progression of liver diseases, including chronic hepatitis $(\mathrm{CH})$, liver cirrhosis (LC), and hepatocellular carcinoma (HCC) $[3,4]$. In addi- tion, meta-analyses have shown that the cytokines have anti-inflammatory effects $[5,6]$. IL- 8 has been studied as one of the most important cytokines in the process of inflammation and immunity of $\operatorname{HCC}[3,7-15]$. IL- 8 may be a potential therapeutic target in the treatment of HCC [16]. Alpha-fetoprotein (AFP) is the most important serum biomarker (as a gold standard) in HCC with a sensitivity of $41 \%$ to $65 \%$ [17]. A number of studies have investigated whether IL- 8 can be an alternative to AFP in patients with HCC. This metaanalysis was designed to assess the serum IL-8 levels in HCC patients compared to healthy controls and patients with $\mathrm{LC}$ and $\mathrm{CH}$. 


\section{Material and methods}

\section{Search strategy}

We systematically searched the terms "IL-8" or "interleukin 8", "liver cancer", "HCC", or "hepatocellular carcinoma", and "serum" in three databases - PubMed (limited to human studies), Scopus (limited to articles), and Web of Science (limited to articles) - up to November 2017 without language restriction in all databases.

\section{Inclusion and exclusion criteria}

The inclusion criteria consisted of studies with English-language abstract reporting the mean/median of serum IL-8 level in HCC patients and healthy controls, human studies, studies reporting the blood sample of IL-8 after overnight fasting and HCC patients without chronic hepatitis or liver cirrhosis. The exclusion criteria included studies with incomplete reports (insufficient information) of the data, conference papers, review studies, animal studies, cross-sectional studies, and studies reporting polymorphisms of IL-8.

\section{Study selection}

One reviewer (M.S.) checked the titles and/or abstracts of each study and then evaluated the full texts of the relevant articles (studies reporting IL-8 levels in HCC patients compared with healthy controls that met the eligibility criteria). The second reviewer (E.S.) re-assessed them.

\section{Data extraction}

One of the reviewers (M.S) extracted the information of all the studies included in the meta-analysis (Table 1).

\section{Statistical analysis}

Review Manager 5.3 (RevMan 5.3) was used to estimate the mean difference (MD) and 95\% confidence interval (CI) in a random-effects model. Q and I-squared $\left(\mathrm{I}^{2}\right)$ statistics were used to estimate heterogeneity across the studies if $p<0.1\left(\mathrm{I}^{2}>50 \%\right)$. In the analyses, a $p$-value $(2$-sided $)<0.05$ showed a statistically significant difference. Subgroup analyses were conducted by comparison of two groups (HCC vs. control,

Table 1. Characteristics of studies included in the meta-analysis $(n=10)$

\begin{tabular}{|c|c|c|c|c|c|c|}
\hline First author, year & Country & $\begin{array}{c}\text { No. of HCC } \\
\text { patients/mean age } \\
\text { (year)/male } \%\end{array}$ & $\begin{array}{l}\text { No. of healthy } \\
\text { controls/mean age } \\
\text { (year)/male } \%\end{array}$ & $\begin{array}{l}\text { No. of } L C \text { patients/ } \\
\text { mean age (year)/ } \\
\text { male } \%\end{array}$ & $\begin{array}{c}\text { No. of } \mathrm{CH} \\
\text { patients } / \text { mean age } \\
\text { (year)/male } \%\end{array}$ & Method (source) \\
\hline Okada, 1994 [7] & Japan & $73 /-/-$ & $20 /-/-$ & $24 /-/-$ & $18 /-/-$ & $\begin{array}{c}\text { ELISA kit (Toray Fuji Bionics, } \\
\text { Tokyo, Japan) }\end{array}$ \\
\hline Ren, 2003 [8] & China & $59 / 50 / 71.2$ & $15 /-/-$ & - & - & $\begin{array}{l}\text { ELISA kits (R\&D Systems, } \\
\text { Minneapolis, MN) }\end{array}$ \\
\hline Ren, 2003a [9] & China & $\begin{array}{l}\text { 55/median: } \\
\text { 51/72.7 }\end{array}$ & $15 /-/-$ & - & - & $\begin{array}{l}\text { ELISA kits (R\&D Systems, } \\
\text { Minneapolis, MN) }\end{array}$ \\
\hline Tachibana, 2007 [10] & Japan & $30 / 66.6 / 60$ & $17 / 58.6 / 70.5$ & 29/58/62.1 & $30 / 48.8 / 76.7$ & Sandwich ELISA \\
\hline Zekri, 2010 [11] & Egypt & $30 / 60.7 /-$ & $9 / 50.9 / 77.8$ & - & - & $\begin{array}{c}\text { ELISA kits (R\&D Systems, Inc. } \\
614 \text { McKinley Place N.E. MN } \\
55413, \text { USA) }\end{array}$ \\
\hline Chao, 2012 [12] & Taiwan & 73/matched/91.8 & $\begin{array}{l}\text { 30/matched/ } \\
\text { matched }\end{array}$ & - & - & $\begin{array}{c}\text { ELISA kits (R\&D Systems Inc., } \\
\text { Minneapolis, MN, USA) }\end{array}$ \\
\hline El-Tayeh, 2012 [13] & Egypt & $\begin{array}{l}\text { 37/median: } \\
\text { 55/78.4 }\end{array}$ & $\begin{array}{l}\text { 16/median: } \\
46 / 68.7\end{array}$ & $\begin{array}{l}\text { 28/median: } \\
\text { 49/78.6 }\end{array}$ & - & ELISA kits \\
\hline Welling, 2012 [14] & USA & $90 / 61 / 76.7$ & - & $180 / 53 / 71.7$ & - & $\begin{array}{l}\text { Bio-Plex kits (Millipore, } \\
\text { Billerica, MA) }\end{array}$ \\
\hline Yahya, 2013 [3] & Egypt & 20/matched/- & 20/matched/65 & - & - & $\begin{array}{c}\text { ELISA kits (Orgenium } \\
\text { Laboratories Business } \\
\text { unit, Finland) }\end{array}$ \\
\hline Zekri, 2015 [15] & Egypt & $192 / 56.7 / 79$ & $95 / 33.4 / 22$ & $96 / 54 / 70$ & - & $\begin{array}{l}\text { ELISA kits (R\&D Systems, } \\
\text { Inc., USA) }\end{array}$ \\
\hline
\end{tabular}

ELISA - enzyme-linked immunosorbent assay, HCC - hepatocellular carcinoma, LC - liver cirrhosis, CH - chronic hepatitis 
HCC vs. liver cirrhotic, and HCC vs. liver hepatitis). Sensitivity analysis was performed as the secondary analysis to evaluate any effect of the individual data on the pooled MDs that we used in two subgroup analyses (patients with HCC patients vs. controls and HCC vs. patients with LC). In addition, both Begg's and Egger's tests were used to evaluate publication bias through funnel plot analysis. We used $\mathrm{pg} / \mathrm{ml}$ as the unit of measurement of serum IL-8.

\section{Results}

A total of 239 studies were found in databases. After removing duplications, 152 studies were screened. Out of all studies screened, 129 studies were excluded and the 23 remaining studies were assessed based on their full text. After that, 13 other studies were omitted with reasons (Fig. 1). Finally, 10 articles were included and analyzed in the meta-analysis.

Table 1 presents some information of the studies included in the meta-analysis. The studies were reported from 1994 to 2015 . Four studies were reported from Egypt [3, 11, 13, 15], two from Japan [7, 10], two from China [8,9], one from Taiwan [12], and one from the USA [14]. The studies had recruited 659 HCC patients, 237 controls, 357 patients with LC, and 48 patients with $\mathrm{CH}$. The mean age, male percentage, and measurement method of IL-8 are shown in Table 1 .

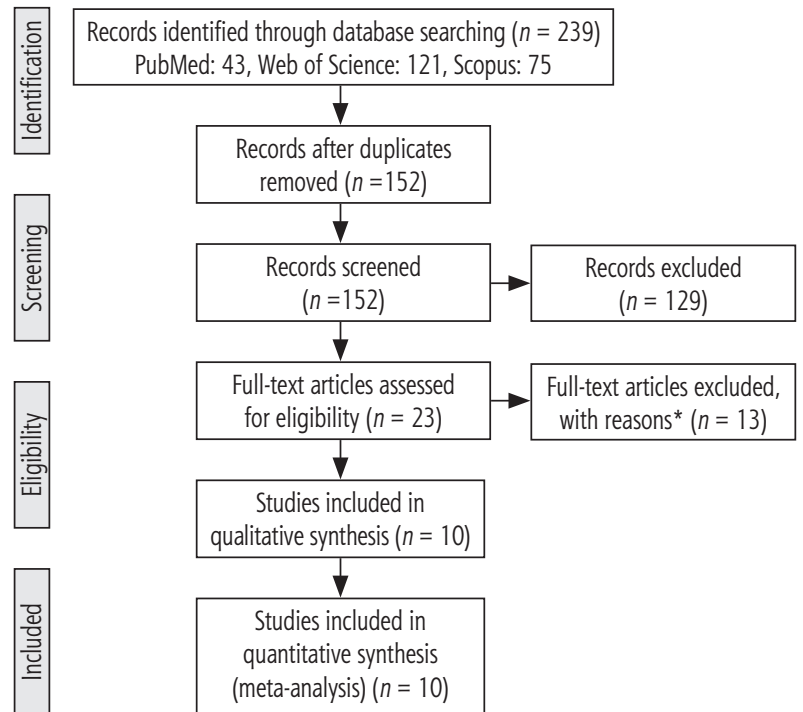

* 2 studies reported the polymorphisms of IL-8, 6 reported studies were cross-sectional studies (just reported HCC group), 1 study was a conference paper, 1 study reported plasma IL-8 level, 1 study did not report mean/median of IL-8 in controls, 1 study reported HCC patients with chronic hepatitis, and 1 study reported HCC patients with liver cirrhosis.

HCC - hepatocellular carcinoma

Fig. 1. Flowchart of the study

The forest plot of serum IL-8 levels in HCC patients compared to healthy controls is shown in Figure 2. The pooled MD for this analysis (569 HCC patients vs. 227 controls) was 39.48 [95\% CI: 152.31, 406.47, $\left.p<0.00001, \mathrm{I}^{2}=98 \%(p<0.00001)\right]$.

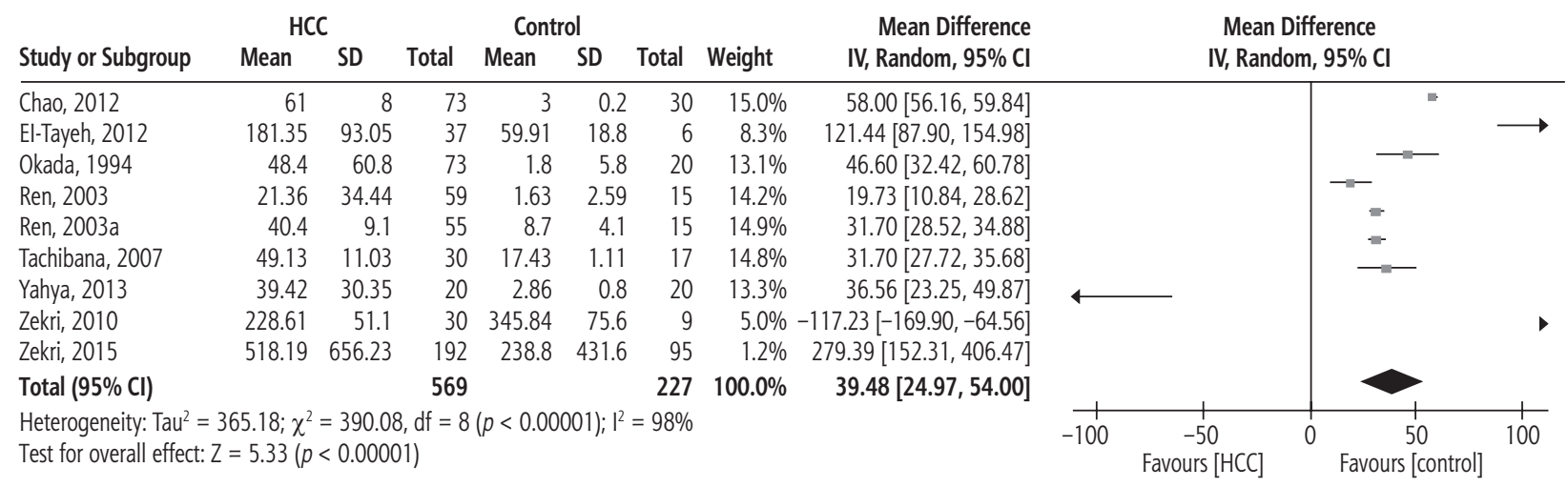

Fig. 2. Forest plot of random effects of serum interleukin 8 (IL-8) levels in hepatocellular carcinoma (HCC) patients compared to healthy controls

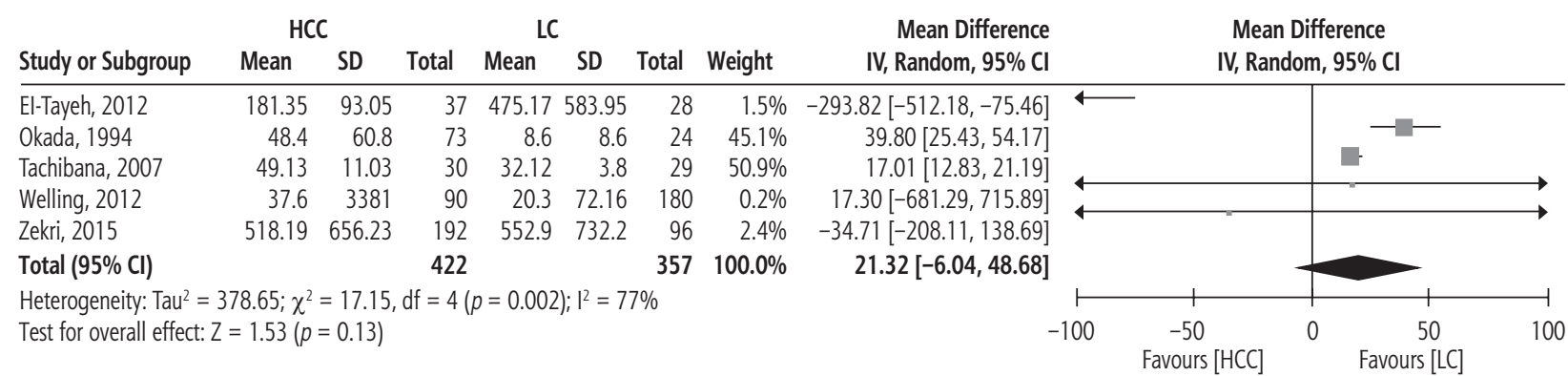

Fig. 3. Forest plot of random effects of serum interleukin 8 (IL-8) levels in hepatocellular carcinoma (HCC) patients compared to patients with liver cirrhosis (LC) 


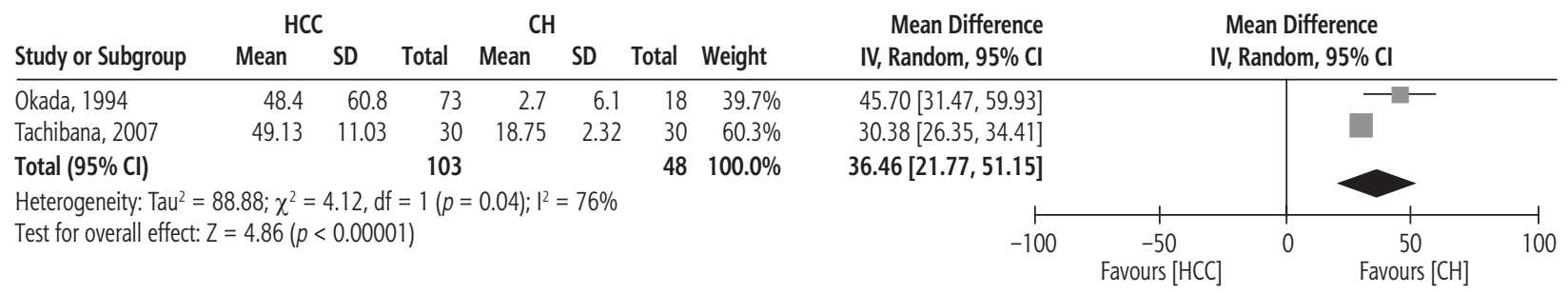

Fig. 4. Forest plot of random effects of serum interleukin 8 (IL-8) levels in hepatocellular carcinoma (HCC) patients compared to patients with chronic hepatitis (CH)
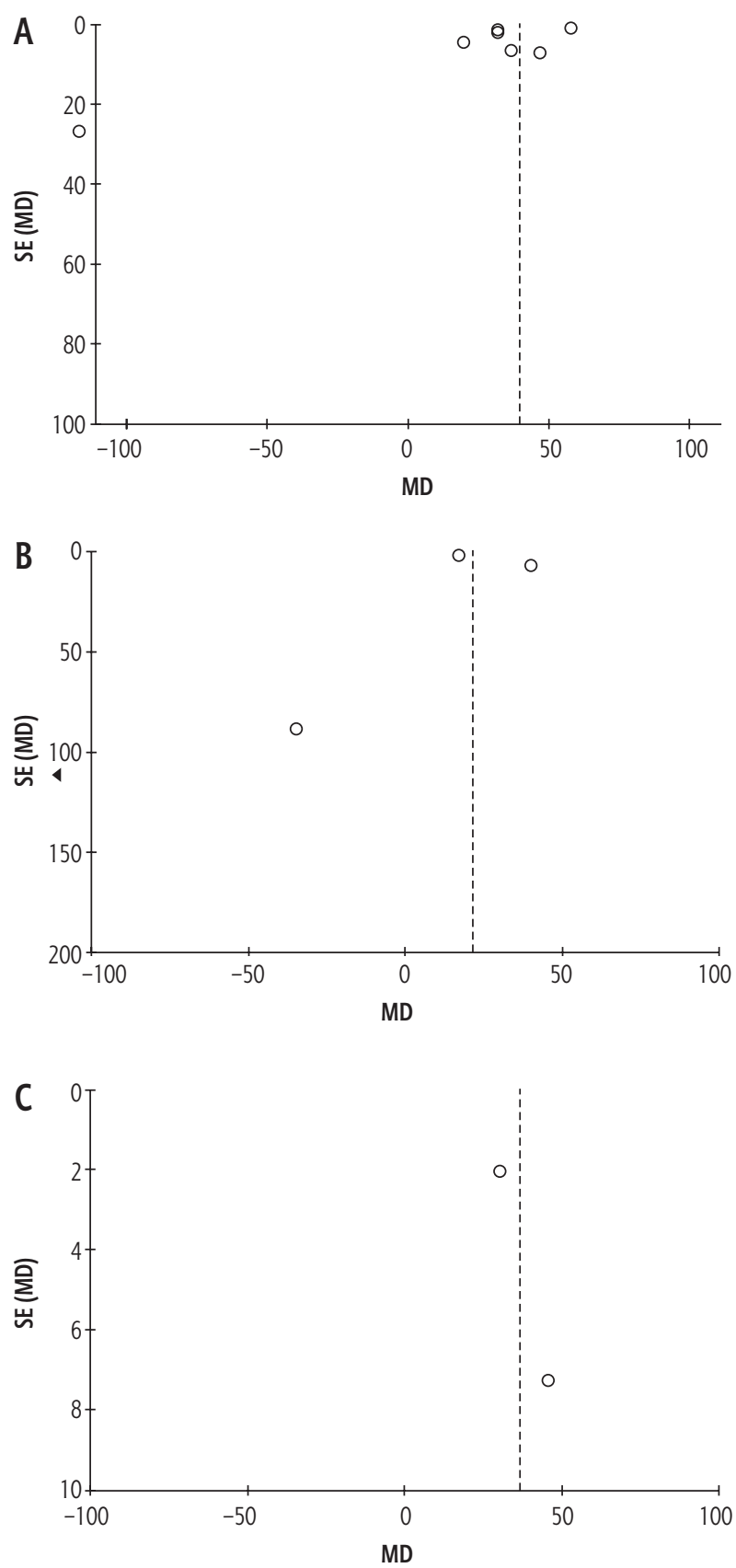

Fig. 5. Funnel plot (random-effects model) of serum interleukin 8 (IL-8) in HCC patients compared to (A) healthy controls, (B) patients with liver cirrhosis (LC) and (C) patients with chronic hepatitis (CH).
Figure 3 illustrates the forest plot of serum IL-8 levels in HCC patients compared to patients with LC. The pooled MD for the analysis (422 HCC patients vs. 357 patients with LC) was 21.32 [95\% CI: $-6.04,48.68$, $\left.p=0.13, I^{2}=77 \%(p=0.002)\right]$.

The forest plot of serum IL-8 levels in HCC patients compared to patients with $\mathrm{CH}$ is shown in Figure 4. The pooled MD for the analysis (103 HCC patients vs. 48 patients with $\mathrm{CH}$ ) was 36.46 [95\% CI: $21.77,51.15$, $\left.p<0.00001, \mathrm{I}^{2}=76 \%(p=0.04)\right]$.

\section{Publication bias}

Figure 5 shows the pooled data of serum IL-8 in three subgroup analyses. Egger's and Begg's tests did not reveal publication bias among the included studies (Figs. 5A and 5B). These tests could not be run on the results of serum IL-8 level in patients with HCC patients compared to patients with $\mathrm{CH}$ because there were less than three studies in this subgroup analysis (Fig. 5C).

\section{Sensitivity analysis}

Sensitivity analysis deleting each study at a time was done following the changes in the pooled MDs and $95 \%$ CIs (Table 2). We deleted the study by Zekri et al. [11] because outlier data in the subgroup analysis of the comparison of serum IL-8 level in patients with HCC patients compared to healthy controls showed that the pooled MD reached 47.31 [95\% CI: $\left.33.06,61.55, p=0.00001, \mathrm{I}^{2}=98 \%\right]$. Also, we deleted the study by El-Tayeh et al. [13] because outlier data in the subgroup analysis of the comparison of serum IL-8 level in the HCC compared to the LC indicated that the pooled MD reached 26.28 [95\% CI: 6.43, 46.12, $\left.p=0.009, \mathrm{I}^{2}=68 \%\right]$.

\section{Discussion}

The results of this meta-analysis showed that the serum IL-8 level in patients with HCC was significantly 
Table 2. Sensitivity analysis for pooled random-effects mean difference (MD) estimates in subgroups

\begin{tabular}{lccccccc}
\hline Subgroup & Removed study & Reason for removal & $Z$ & $p$ & Heterogeneity & MD & $95 \%$ Cl (min, max) \\
\hline HCC vs. control & Zekri, 2010 [11] & Outlier study & 6.51 & $<0.00001$ & $98 \%$ & 47.31 & $33.06,61.55$ \\
\hline HCC vs. LC & El-Tayeh, 2012 [13] & Outlier study & 2.60 & 0.009 & $68 \%$ & 26.28 & $6.43,46.12$ \\
\hline
\end{tabular}

higher than in other groups (healthy controls, and patients with LC and $\mathrm{CH})$. Studies [3, 7-10, 12, 13] have shown a highly significant level of IL-8 in the serum of HCC patients compared to healthy controls, except one study [11]. In addition, out of five studies [7, 10, 13-15], three studies reported significant differences between HCC patients and cirrhotic patients (lowered level in one study and elevated level in two studies), and out of two studies $[13,15]$ reporting serum IL-8 level in patients with HCC compared to patients with $\mathrm{CH}$, both studies reported an elevated level of IL-8.

IL-8 contributes to human malignancies such as HCC and plays its role through participation in the vascular invasion and angiogenesis or tumor proliferation [18]. Attention to IL-8 can control angiogenesis and HCC invasion [8], and IL acts in favor of progressive inflammation, permanent cell damage, and $\mathrm{HCV}$ infection [19]. One study [15] reported that the accuracy of diagnosis of IL-8 was poor for rapid diagnosis of HCC. HCV infection is an important reason for the progression of LC and HC [20], suggesting the direct and indirect roles of $\mathrm{HCV}$ in the pathogenesis of HCC [21]. One study [3] showed that patients with HCV infection had a higher level of IL-8 compared with control subjects, which is in agreement with another study [22], and HCC patients with or without cirrhosis had an elevated level of IL-8 compared to patients with HCV infection [3]. Also, serum IL-8 levels are associated with cirrhosis, and therefore based on the previous results, HCV can activate the IL- 8 promoter and HCV-E2 upregulates the production of IL-8 [22]. Serum IL-8 levels rose as the disease developed from $\mathrm{CH}$ to $\mathrm{LC}$ and then to HCC, which could indicate that increased potency might lead not only to the immune response against $\mathrm{HCV}$ infection but also to the progression of HCC [23].

Alpha-fetoprotein (AFP) is the single most significant tumor marker for HCC. One major mistake is to assume that AFP is critical for early detection of cancer [24]. El-Tayeh et al. [13] recommended that AFP for HCC has a low sensitivity, and IL- 8 is suggested to be evaluated simultaneously to obtain the HCC diagnosis. All forms of AFP enhanced the baseline expression of cytokines [25]. IL-8 is approximately the same as the predictor of AFP in determining the presence of HCC [14] and independently predicts the survival rate in HCC patients, especially in patients with early
HCC $[8,14]$. One study showed a positive correlation between tumor size and IL-8 in HCC patients, while the serum AFP level was not related to the tumor size [18]. Limitations of the included studies in the meta-analysis were: 1) the relationship of $\mathrm{HCV}$ and $\mathrm{HBV}$ with HCC in some studies, 2) the mean age and sex mismatch between the groups in most studies; 3 ) the low number of participants in most studies, and 4) the difference in AFP levels and Child-Pugh score. These differences caused high heterogeneity among the studies in this meta-analysis. However, regarding the high heterogeneity, there was no publication bias in the subgroup analyses.

\section{Conclusions}

An elevated level of serum IL-8 in the HCC patients compared to the three other groups (healthy controls, cirrhotic patients, and patients with $\mathrm{CH}$ ) can show the increased risk for this cytokine in HCC patients. Therefore, the cytokine can be used as a new biomarker replacing AFP or as a clinical assay for evaluation of the pathogenesis and probably the progression or development of HCC. Further studies with a larger number of participants and control of factors involved in $\mathrm{HCC}$ are needed to evaluate the correlation between IL- 8 and AFP to achieve a better and more accurate conclusion.

\section{Disclosure}

Authors report no conflict of interest.

\section{References}

1. Rahbari NN, Mehrabi A, Mollberg NM, et al. Hepatocellular carcinoma: current management and perspectives for the future. Ann Surg 2011; 253: 453-469.

2. Jang JW, Oh BS, Kwon JH, et al. Serum interleukin-6 and C-reactive protein as a prognostic indicator in hepatocellular carcinoma. Cytokine 2012; 60: 686-693.

3. Yahya RS, Ghanem OH, Foyouh AA, et al. Role of interleukin-8 and oxidative stress in patients with hepatocellular carcinoma. Clin Lab 2013; 59: 969-976.

4. Polyak SJ, Khabar KS, Paschal DM, et al. Hepatitis C virus nonstructural 5 A protein induces interleukin-8, leading to partial inhibition of the interferon-induced antiviral response. J Virol 2001; 75: 6095-6106.

5. Mozaffari HR, Sharifi R, Sadeghi M. Interleukin-6 levels in the serum and saliva of patients with oral lichen planus compared 
with healthy controls: a meta-analysis study. Cent Eur J Immunol 2018; 43: 103-108.

6. Mozaffari HR, Ramezani M, Mahmoudiahmadabadi M, et al. Salivary and serum levels of tumor necrosis factor-alpha in oral lichen planus: a systematic review and meta-analysis study. Oral Surg Oral Med Oral Pathol Oral Radiol 2017; 124: e183-e189.

7. Okada K, Shimizu Y, Tsukishiro T, et al. Serum interleukin-8 levels in patients with hepatocellular carcinoma. Int Hepatol Commun 1994; 2: 178-182.

8. Ren Y, Poon RT, Tsui HT, et al. Interleukin-8 serum levels in patients with hepatocellular carcinoma: correlations with clinicopathological features and prognosis. Clin Cancer Res 2003; 9: 5996-6001.

9. Ren Y, Tsui HT, Poon RT, et al. Macrophage migration inhibitory factor: roles in regulating tumor cell migration and expression of angiogenic factors in hepatocellular carcinoma. Int J Cancer 2003a; 107: 22-29.

10. Tachibana Y, Nakamoto Y, Mukaida N, et al. Intrahepatic interleukin-8 production during disease progression of chronic hepatitis C. Cancer Lett 2007; 251: 36-42.

11. Zekri AR, Alam El-Din HM, Bahnassy AA, et al. Serum levels of soluble Fas, soluble tumor necrosis factor-receptor II, interleukin-2 receptor and interleukin-8 as early predictors of hepatocellular carcinoma in Egyptian patients with hepatitis $\mathrm{C}$ virus genotype-4. Comp Hepatol 2010; 9: 1.

12. Chao Y, Wu CY, Kuo CY, et al. Cytokines are associated with postembolization fever and survival in hepatocellular carcinoma patients receiving transcatheter arterial chemoembolization. Hepatol Int 2013; 7: 883-892.

13. El-Tayeh SF, Hussein TD, El-Houseini ME, et al. Serological biomarkers of hepatocellular carcinoma in Egyptian patients. Dis Markers 2012; 32: 255-263.

14. Welling TH, Fu S, Wan S, et al. Elevated serum IL-8 is associated with the presence of hepatocellular carcinoma and independently predicts survival. Cancer Invest 2012; 30: 689-697.

15. Zekri AR, Youssef AS, Bakr YM, et al. Serum biomarkers for early detection of hepatocellular carcinoma associated with HCV infection in egyptian patients. Asian Pac J Cancer Prev 2015; 16: 1281-1287.

16. Park SY, Han J, Kim JB, et al. Interleukin-8 is related to poor chemotherapeutic response and tumourigenicity in hepatocellular carcinoma. Eur J Cancer 2014; 50: 341-350.

17. Gupta S, Bent S, Kohlwes J. Test characteristics of alpha-fetoprotein for detecting hepatocellular carcinoma in patients with hepatitis C. A systematic review and critical analysis. Ann Intern Med 2003; 139: 46-50.

18. Kubo F, Ueno S, Hiwatashi K, et al. Interleukin 8 in human hepatocellular carcinoma correlates with cancer cell invasion of vessels but not with tumor angiogenesis. Ann Surg Oncol 2005; 12: 800-807.

19. Fan XG, Liu WE, Li CZ, et al. Circulating Th1 and Th2 cytokines in patients with hepatitis $C$ virus infection. Mediators Inflamm 1998; 7: 295-297.

20. Seeff LB. Natural history of chronic hepatitis C. Hepatology 2002; 36: S35-46.

21. Tran G. The role of hepatitis $C$ virus in the pathogenesis of hepatocellular carcinoma. Bioscience Horizons 2008; 1: 167-175.

22. Mihm U, Herrmann E, Sarrazin U, et al. Association of serum interleukin-8 with virologic response to antiviral therapy in patients with chronic hepatitis C. J Hepatol 2004; 40: 845-852.

23. Polyak SJ, Khabar KSA, Rezeiq M, et al. Elevated levels of interleukin-8 in serum are associated with hepatitis $C$ virus infection and resistance to interferon therapy. J Virol 2001; 75: 6209-6011.
24. Zakhary NI, El-Merzabani MM, El-Sawi NM, et al. Impact of different biochemical markers in serum of patients with benign and malignant liver diseases. J Adv Res 2011; 2: 49-55.

25. Potapovich AI, Pastore S, Kostyuk VA, et al. alpha-Fetoprotein as a modulator of the pro-inflammatory response of human keratinocytes. Br J Pharmacol 2009; 158: 1236-1247. 